\title{
GLOBAL SMALL SOLUTIONS OF HEAT CONDUCTIVE COMPRESSIBLE NAVIER-STOKES EQUATIONS WITH VACUUM: SMALLNESS ON SCALING INVARIANT QUANTITY
}

\author{
JINKAI LI
}

\begin{abstract}
In this paper, we consider the Cauchy problem to the heat conductive compressible Navier-Stokes equations in the presence of vacuum and with vacuum far field. Global well-posedness of strong solutions is established under the assumption, among some other regularity and compatibility conditions, that the scaling invariant quantity $\left\|\rho_{0}\right\|_{\infty}\left(\left\|\rho_{0}\right\|_{3}+\left\|\rho_{0}\right\|_{\infty}^{2}\left\|\sqrt{\rho_{0}} u_{0}\right\|_{2}^{2}\right)\left(\left\|\nabla u_{0}\right\|_{2}^{2}+\left\|\rho_{0}\right\|_{\infty}\left\|\sqrt{\rho_{0}} E_{0}\right\|_{2}^{2}\right)$ is sufficiently small, with the smallness depending only on the parameters $R, \gamma, \mu, \lambda$, and $\kappa$ in the system. The total mass can be either finite or infinite.
\end{abstract}

\section{INTRODUCTION}

In this paper, we consider the following heat conductive compressible Navier-Stokes equations for the ideal gas:

$$
\begin{array}{r}
\partial_{t} \rho+\operatorname{div}(\rho u)=0, \\
\rho\left(\partial_{t} u+(u \cdot \nabla) u\right)-\mu \Delta u-(\mu+\lambda) \nabla \operatorname{div} u+\nabla p=0, \\
c_{v} \rho\left(\partial_{t} \theta+u \cdot \nabla \theta\right)+p \operatorname{div} u-\kappa \Delta \theta=\mathcal{Q}(\nabla u),
\end{array}
$$

in $\mathbb{R}^{3} \times(0, \infty)$, where the unknowns $\rho \geq 0, u \in \mathbb{R}^{3}$, and $\theta \geq 0$, respectively, represent the density, velocity, and absolute temperature, $p=R \rho \theta$, with positive constant $R$, is the pressure, $c_{v}>0$ is a constant, constants $\mu$ and $\lambda$ are the bulk and shear viscous coefficients, respectively, positive constant $\kappa$ is the heat conductive coefficient, and

$$
\mathcal{Q}(\nabla u)=\frac{\mu}{2}\left|\nabla u+(\nabla u)^{t}\right|^{2}+\lambda(\operatorname{div} u)^{2},
$$

with $(\nabla u)^{t}$ being the transpose of $\nabla u$. The viscous coefficients $\mu$ and $\lambda$ satisfy the physical constraints

$$
\mu>0, \quad 2 \mu+3 \lambda \geq 0 .
$$

The additional assumption $2 \mu>\lambda$ will also be use in this paper.

Due to their fundamental importance in the fluid dynamics, extensive studies have been carried out and many developments have been achieved on the compressible

Date: June 21, 2019.

2010 Mathematics Subject Classification. 35A01, 35Q30, 35Q35, 76N10.

Key words and phrases. Heat conductive compressible Navier-Stokes equations; global wellposedness; strong solutions. 
Navier-Stokes equations in the last seventy years. The mathematical studies on the compressible Navier-Stokes equations started with the uniqueness results by Graffi [18] in 1953 for barotropic fluid and by Serrin [51] in 1959 for general fluids, and the local existence result by Nash [49] in 1962 for the Cauchy problem. Since then, comprehensive mathematical theories have been developed for the compressible NavierStokes equations.

The mathematical theory for the compressible Navier-Stokes equations in 1D is satisfied and, in particular, the corresponding global well-posedness, for arbitrary large initial data, and the initial density can either be uniformly positive or only nonnegative (that is, it can vanish on some subset of the domain). For the case that the initial density is uniformly positive, the global well-posedness of strong solutions, with large initial data, was first proved in [28], for the isentropic case, and later in [30], for the general case, and the corresponding large time behavior was recently proved in [36], see also [2, 27, 29, 61, 62] for some related results. For the case that the initial density contains vacuum, the corresponding global well-posedness of strong solutions was recently proved by the author and his collaborator, see [35, 38, 39].

Compared with the one dimensional case, the mathematical theory for the multidimensional case is far from satisfied and, in particular, some basic problems such as the global existence of strong solutions and uniqueness of weak solution are still unknown. For the case that the initial density is uniformly positive, the local wellposedness was proved long time ago, see [24, 43, 49, 52, 53, 55] and, in particular, the inflow and outflow were allowed in [43]; however, the general global well-posedness is still unknown. Global well-posedness of strong solutions with small initial data was first proved in [44 [47], and later further developed in many papers, see, e.g., [3, 7, 11 14, 19, 31, 50, 54]. For the case that the initial density allows vacuum, global existence of weak solutions was first proved in [41, 42], see [1, 15 17, 26] for further developments, but the uniqueness is still an open problem. Local well-posedness of strong solutions was proved in [8 10], and the global well-posedness, with small initial data, was proved in [22], and see [21, 37, 58] for further developments.

The aim of this paper is to establish the global existence of strong solutions to the Cauchy problem of (1.1)-(1.3), under some smallness assumptions on the initial data, in the presence of initial vacuum, and with vacuum far field. The main novelty of this paper is that the smallness assumption is imposed on some quantities that are scaling invariant with respect to the following scaling transformation:

$$
\left(\rho_{0 \lambda}(x), u_{0 \lambda}(x), \theta_{0 \lambda}(x)\right)=\left(\rho_{0}(\lambda x), \lambda u_{0}(\lambda x), \lambda^{2} \theta_{0}(\lambda x)\right), \quad \forall \lambda \neq 0 .
$$

This scaling transformation on the initial data inheres in the following natural scaling invariant property of system (1.1)-(1.3):

$$
\rho_{\lambda}(x, t)=\rho\left(\lambda x, \lambda^{2} t\right), \quad u_{\lambda}(x, t)=\lambda u\left(\lambda x, \lambda^{2} t\right), \quad \theta_{\lambda}(x, t)=\lambda^{2} \theta\left(\lambda x, \lambda^{2} t\right),
$$

that is, if $(\rho, u, \theta)$ is a solution, with initial data $\left(\rho_{0}, u_{0}, \theta_{0}\right)$, then $\left(\rho_{\lambda}, u_{\lambda}, \theta_{\lambda}\right)$ is also a solution, for any nonzero $\lambda$, but with initial data $\left(\rho_{0 \lambda}, u_{0 \lambda}, \theta_{0 \lambda}\right)$. 
The reason for us to focus on the smallness assumptions on the scaling invariant quantities, rather than on those not, is the following fact: if assuming that $\mathscr{M}$ is a functional, satisfying

$$
\mathscr{M}\left(\rho_{0 \lambda}, u_{0 \lambda}, \theta_{0 \lambda}\right)=\lambda^{\ell}\left(\rho_{0}, u_{0}, \theta_{0}\right), \quad \forall \lambda \neq 0, \quad \text { for some constant } \ell \neq 0,
$$

and that the global well-posedness holds, for any initial data $\left(\rho_{0}, u_{0}, \theta_{0}\right)$, such that $\mathscr{M}\left(\rho_{0}, u_{0}, \theta_{0}\right) \leq \varepsilon_{0}$, for some $\varepsilon_{0}>0$ depending only on the parameters of the system, then, by suitably choosing the scaling parameter $\lambda$, one can show that the system is actually globally well-posed, for arbitrary large initial data; however, this global well-posedness for arbitrary large initial data is far from what we already known.

Before stating the main results, we first clarify some necessary notations being used throughout this paper. For $1 \leq q \leq \infty$ and positive integer $m$, we use $L^{q}=$ $L^{q}\left(\mathbb{R}^{3}\right)$ and $W^{1, q}=W^{m, q}\left(\mathbb{R}^{3}\right)$ to denote the standard Lebesgue and Sobolev spaces, respectively, and in the case that $q=2$, we use $H^{m}$ instead of $W^{m, 2}$. For simplicity, we also use notations $L^{q}$ and $H^{m}$ to denote the $N$ product spaces $\left(L^{q}\right)^{N}$ and $\left(H^{m}\right)^{N}$, respectively. We always use $\|u\|_{q}$ to denote the $L^{q}$ norm of $u$. For shortening the expressions, we sometimes use $\left\|\left(f_{1}, f_{2}, \cdots, f_{n}\right)\right\|_{X}$ to denote the sum $\sum_{i=1}^{N}\left\|f_{i}\right\|_{X}$ or its equivalent norm $\left(\sum_{i=1}^{N}\left\|f_{i}\right\|_{X}^{2}\right)^{\frac{1}{2}}$. We denote

$$
\begin{gathered}
D^{k, r}=\left\{u \in L_{l o c}^{1}\left(\mathbb{R}^{3}\right) \mid\left\|\nabla^{k} u\right\|_{r}<\infty\right\}, \quad D^{k}=D^{k, 2}, \\
D_{0}^{1}=\left\{u \in L^{6} \mid\|\nabla u\|_{2}<\infty\right\} .
\end{gathered}
$$

For simplicity of notations, we adopt the notation

$$
\int f d x=\int_{\mathbb{R}^{3}} f d x
$$

We are now ready to state the main result of this paper.

Theorem 1.1. Assume $2 \mu>\lambda$ and let $q \in(3,6]$ be a fixed constant. Assume that the initial data $\left(\rho_{0}, u_{0}, \theta_{0}\right)$ satisfies

$$
\begin{gathered}
\rho_{0}, \theta_{0} \geq 0, \quad \rho \leq \bar{\rho}, \quad \rho_{0} \in H^{1} \cap W^{1, q}, \quad \sqrt{\rho_{0}} \theta_{0} \in L^{2}, \quad\left(u_{0}, \theta_{0}\right) \in D_{0}^{1} \cap D^{2}, \\
-\mu \Delta u_{0}-(\mu+\lambda) \nabla d i v u_{0}+\nabla p_{0}=\sqrt{\rho_{0}} g_{1}, \quad \kappa \Delta \theta_{0}+\mathcal{Q}(\nabla u)=\sqrt{\rho_{0}} g_{2},
\end{gathered}
$$

for a positive constant $\bar{\rho}$ and some $\left(g_{1}, g_{2}\right) \in L^{2}$, where $p_{0}=R \rho_{0} \theta_{0}$.

Then, there is a positive number $\varepsilon_{0}$ depending only on $R, \gamma, \mu, \lambda$, and $\kappa$, such that system (1.1)-(1.3), with initial data $\left(\rho_{0}, u_{0}, \theta_{0}\right)$, has a unique global solution $(\rho, u, \theta)$, satisfying

$$
\begin{gathered}
\rho \in C\left([0, \infty) ; H^{1} \cap W^{1, q}\right), \quad \rho_{t} \in C\left([0, \infty) L^{2} \cap L^{q}\right), \\
(u, \theta) \in C\left([0, \infty) ; D_{0}^{1} \cap D^{2}\right) \cap L_{l o c}^{2}\left([0, \infty) ; D^{2, q}\right), \\
\left(u_{t}, \theta_{t}\right) \in L_{l o c}^{2}\left([0, \infty) ; D_{0}^{1}\right), \quad\left(\sqrt{\rho} u_{t}, \sqrt{\rho} \theta_{t}\right) \in L_{l o c}^{\infty}\left([0, \infty) ; L^{2}\right),
\end{gathered}
$$


provided

$$
\mathscr{N}_{0}:=\bar{\rho}\left(\left\|\rho_{0}\right\|_{3}+\bar{\rho}^{2}\left\|\sqrt{\rho_{0}} u_{0}\right\|_{2}^{2}\right)\left(\left\|\nabla u_{0}\right\|_{2}^{2}+\bar{\rho}\left\|\sqrt{\rho_{0}} E_{0}\right\|_{2}^{2}\right) \leq \varepsilon_{0} .
$$

Remark 1.1. (i) One can easily check that the quantity $\mathscr{N}_{0}$ in Theorem 1.1 is scaling invariant, with respect to this scaling transformation (1.4). Therefore, Theorem 1.1 provides the global well-posedness of system (1.1)-(1.3) under some smallness assumption on a scaling invariant quantity, for the case that the vacuum is allowed.

(ii) Global well-posedness of strong solutions to the Cauchy problem of system (1.1) - (1.3) in the presence of vacuum has been proved in [21] and [58], with nonvacuum far field and vacuum far field, respectively. The assumptions concerning the smallness in [21] and [58] are imposed as

$$
\begin{aligned}
C_{0} & =\int\left(\frac{\rho_{0}}{2}\left|u_{0}\right|^{2}+R\left(\rho_{0} \log \rho_{0}-\rho_{0}+1\right)+\frac{R}{\gamma-1} \rho_{0}\left(\theta_{0}-\log \theta_{0}+1\right)\right) d x \\
& \leq \varepsilon_{0}=\varepsilon_{0}\left(\left\|\rho_{0}\right\|_{\infty},\left\|\theta_{0}\right\|_{\infty},\left\|\nabla u_{0}\right\|_{2}, R, \gamma, \mu, \lambda, \kappa\right)
\end{aligned}
$$

and

$$
\int \rho_{0} d x \leq \varepsilon_{0}=\varepsilon_{0}\left(\left\|\rho_{0}\right\|_{\infty},\left\|\sqrt{\rho_{0}} \theta_{0}\right\|_{2},\left\|\nabla u_{0}\right\|_{2}, R, \gamma, \mu, \lambda, \kappa\right),
$$

respectively. However, since the explicit dependence of $\varepsilon_{0}$ on $\left\|\rho_{0}\right\|_{\infty},\left\|\theta_{0}\right\|_{\infty},\left\|\sqrt{\rho_{0}} \theta_{0}\right\|_{2}$, and $\left\|\nabla u_{0}\right\|_{2}$ are not derived in [21, 58], the scaling invariant quantities, on which the smallness guarantees the global well-posedness, can not be identified there.

(iii) Comparing with the global well-posedness result in [58], our result, Theorem 1.1, allows the initial mass to be infinite. This will be crucial for obtaining the global entropy-bounded solutions in our forthcoming paper [40].

Comparing with the isentropic case considered in [22], the additional difficulty for studying the global well-posedness of the full compressible Navier-Stokes equations is that the following basic energy inequality does not provide any dissipation estimates:

$$
\int \rho\left(\frac{|u|^{2}}{2}+c_{v} \theta\right) d x=\int \rho_{0}\left(\frac{\left|u_{0}\right|^{2}}{2}+c_{v} \theta_{0}\right) d x .
$$

Note that the dissipation estimates of the form $\int_{0}^{T}\|\nabla u\|_{2}^{2} \leq C$, which can be guaranteed by the basic energy estimates for the isentropic case, is crucial in the arguments of [22]. To overcome this difficulty, some kinds of dissipative estimates were recovered for the full compressible Navier-Stokes equations in [21] and [58], for the cases that with non-vacuum and vacuum far field, respectively, by using the entropy inequality and the conservation of mass. Notcing that the entropy inequality, one of the keys in [21], holds only for the case that with non-vacuum far field, and the finiteness of the mass is required in [58], and recalling that we consider the case that with vacuum far field and allowing possible infinite mass, the arguments in [21, 58] do not work for our case. 
A crucial ingredient of obtaining the dissipative estimates is the following new equation (see the proof in Proposition 2.4)

$$
\frac{2 \mu+\lambda}{2}\left(\partial_{t} \rho^{3}+\operatorname{div}\left(u \rho^{3}\right)\right)+\rho^{3} p+\rho^{3} \Delta^{-1} \operatorname{div}(\rho u)_{t}+\rho^{3} \Delta^{-1} \operatorname{div} \operatorname{div}(\rho u \otimes u)=0,
$$

which is derived by combining the continuity equation and the momentum equation; note that the temperature equation plays no role in deriving this. Comparing with the continuity equation, the main advantage of the above equation is that it enables us to get $L^{\infty}\left(0, T ; L^{3}\right)$ estimate of $\rho$ without appealing to the the $L^{\infty}$ of $\operatorname{div} u$. In fact, the above equation leads to the following kind of inequality

$$
\sup _{0 \leq t \leq T}\|\rho\|_{3}^{3}+\int_{0}^{T} \int \rho^{3} p d x d t \lesssim \sup _{0 \leq t \leq T}\left(\|\rho\|_{\infty}^{\frac{2}{3}}\|\sqrt{\rho} u\|_{2}^{\frac{1}{3}}\left\|\sqrt{\rho}|u|^{2}\right\|_{2}^{\frac{1}{3}}\|\rho\|_{3}^{3}\right)+\cdots,
$$

see Proposition 2.4 for the details. This motivates us to impose the smallness conditions on $\left\|\rho_{0}\right\|_{\infty}^{2}\left\|\sqrt{\rho_{0}} u_{0}\right\|_{2}\left\|\sqrt{\rho_{0}}\left|u_{0}\right|^{2}\right\|_{2}$ (this is one of the terms of $\mathscr{N}_{0}$ in Theorem 1.1) to get the bound of $\|\rho\|_{3}$. The above inequality also guides us to carry out the estimates on $\|\sqrt{\rho} u\|_{L^{\infty}\left(0, T ; L^{2}\right)},\|\sqrt{\rho} E\|_{L^{\infty}\left(0, T ; L^{2}\right)}$, and $\|\rho\|_{L^{\infty}\left(0, T ; L^{\infty}\right)}$, which are performed in Propositions 2.2, 2.3, and 2.6, respectively. Higher order estimates are required in the estimate for $\|\rho\|_{L^{\infty}\left(0, T ; L^{\infty}\right)}$, and they are carried out with the help of $\omega=\nabla \times u$ and $G=(2 \mu+\lambda) \operatorname{div} u-p$, which turn out to have better properties than $\nabla u$, see Proposition 2.5, Combining Proposition 2.2, 2.3, 2.4, 2.6, and 2.5, by continuity arguments, we are able to get time-independent estimate on the scaling invariant quantity $\mathscr{N}_{T}$, under the condition that $\mathscr{N}_{0}$ is sufficiently small. With this a priori estimate for $\mathscr{N}_{T}$, one can further get the time-independent a priori estimates of $\|\nabla u\|_{L^{\infty}\left(0, T ; L^{2}\right)}$ and $\|\rho\|_{L^{\infty}\left(0, T ; L^{\infty}\right)}$, based on which, the blow-up criteria apply, and, thus, the global well-posedness follows.

Throughout this paper, we use $C$ to denote a general positive constant which may vary from line to line. $A \lesssim B$ means $A \leq C B$ for some positive constant $C$.

\section{A PRIORI ESTIMATES}

This section is devoted to deriving some a priori estimates for the solutions to the Cauchy problem of system (1.1)-(1.3). The existence of solution is guaranteed by the following local well-posedness result proved in [10]:

Proposition 2.1. Under the conditions in Theorem 1.1, there is a positive time $T_{*}$, such that system (1.1)-(1.3), with initial data $\left(\rho_{0}, u_{0}, \theta_{0}\right)$, has a unique solution $(\rho, u, \theta)$, on $\mathbb{R}^{3} \times\left(0, T_{*}\right)$, satisfying

$$
\begin{gathered}
\rho \in C\left(\left[0, T_{*}\right] ; H^{1} \cap W^{1, q}\right), \quad \rho_{t} \in C\left(\left[0, T_{*}\right] L^{2} \cap L^{q}\right), \\
(u, \theta) \in C\left(\left[0, T_{*}\right] ; D_{0}^{1} \cap D^{2}\right) \cap L^{2}\left(0, T_{*} ; D^{2, q}\right), \\
\left(u_{t}, \theta_{t}\right) \in L^{2}\left(0, T_{*} ; D_{0}^{1}\right), \quad\left(\sqrt{\rho} u_{t}, \sqrt{\rho} \theta_{t}\right) \in L_{l o c}^{\infty}\left(0, T_{*} ; L^{2}\right) .
\end{gathered}
$$


In the rest of this section, we always assume that $(\rho, u, \theta)$, is a solution to system (1.1) -(1.3), on $\mathbb{R}^{3} \times(0, T)$, for some positive time $T$, satisfying the regularities in Proposition 2.1 with $T_{*}$ there replaced by $T$, with initial data $\left(\rho_{0}, u_{0}, \theta_{0}\right)$.

\subsection{Energy inequalities.}

Proposition 2.2. The following estimate holds:

$$
\sup _{0 \leq t \leq T}\|\sqrt{\rho} u\|_{2}^{2}+\int_{0}^{T}\|\nabla u\|_{2}^{2} d t \leq C\left\|\sqrt{\rho_{0}} u_{0}\right\|_{2}^{2}+C \int_{0}^{T}\|\rho\|_{3}^{2}\|\nabla \theta\|_{2}^{2} d t,
$$

for a positive constant $C$ depending only on $R, \gamma, \mu, \lambda$, and $\kappa$.

Proof. Multiplying (1.2) by $u$, integration the resultant over $\mathbb{R}^{3}$, and noticing that $\mu+\lambda>0$, it follows from integration by parts and the Cauchy inequality that

$$
\begin{aligned}
& \frac{d}{d t}\|\sqrt{\rho} u\|_{2}^{2}+\mu\|\nabla u\|_{2}^{2}+(\mu+\lambda)\|\operatorname{div} u\|_{2}^{2} \\
\leq & R\|\rho\|_{3}\|\theta\|_{6}\|\operatorname{div} u\|_{2} \leq C\left\|_{\rho}\right\|_{3}\|\nabla \theta\|_{2}\|\operatorname{div} u\|_{2} \\
\leq & (\mu+\lambda)\|\operatorname{div} u\|_{2}^{2}+C\|\rho\|_{3}^{2}\|\nabla \theta\|_{2}^{2},
\end{aligned}
$$

from which, the conclusion follows by integrating in $t$.

Proposition 2.3. Assume that $2 \mu>\lambda$. Then, the following estimate holds:

$$
\begin{aligned}
& \sup _{0 \leq t \leq T}\|\sqrt{\rho} E\|_{2}^{2}+\int_{0}^{T}\left(\|\nabla \theta\|_{2}^{2}+\||u| \nabla u\|_{2}^{2}\right) d t \\
\leq & C\left\|\sqrt{\rho_{0}} E_{0}\right\|_{2}^{2}+C \int_{0}^{T}\|\rho\|_{\infty}\|\rho\|_{3}^{\frac{1}{2}}\|\sqrt{\rho} \theta\|_{2}\|(\nabla \theta,|u| \nabla u)\|_{2}^{2} d t,
\end{aligned}
$$

for a positive constant $C$ depending only on $R, \gamma, \mu, \lambda$, and $\kappa$, where $E=\frac{|u|^{2}}{2}+c_{v} \theta$.

Proof. One can verify

$$
\rho\left(\partial_{t} E+u \cdot \nabla E\right)+\operatorname{div}(u p)-\kappa \Delta \theta=\operatorname{div}(\mathcal{S} \cdot u),
$$

where $\mathcal{S}=\mu\left(\nabla u+(\nabla u)^{t}\right)+\lambda \operatorname{div} u I$. Multiplying (2.1) by $E$, integrating the resultant over $\mathbb{R}^{3}$, it follows from integration by parts that

$$
\begin{aligned}
& \frac{1}{2} \frac{d}{d t}\|\sqrt{\rho} E\|_{2}^{2}+\kappa c_{v}\|\nabla \theta\|_{2}^{2} \\
= & \int\left[-\frac{\kappa}{2} \nabla \theta \cdot \nabla|u|^{2}+(u p-\mathcal{S} \cdot u) \cdot\left(c_{v} \nabla \theta+\frac{\nabla|u|^{2}}{2}\right)\right] d x \\
\leq & \frac{c_{v} \kappa}{2}\|\nabla \theta\|_{2}^{2}+C\||u| \nabla u\|_{2}^{2}+C \int \rho^{2} \theta^{2}|u|^{2} d x,
\end{aligned}
$$

which yields

$$
\frac{d}{d t}\|\sqrt{\rho} E\|_{2}^{2}+\kappa c_{v}\|\nabla \theta\|_{2}^{2} \lesssim\||u| \nabla u\|_{2}^{2}+\int \rho^{2} \theta^{2}|u|^{2} d x
$$


Multiplying (1.2) by $|u|^{2} u$, integrating the resultant over $\mathbb{R}^{3}$, it follows from integration by parts that

$$
\begin{aligned}
\frac{1}{4} \frac{d}{d t}\left\|\sqrt{\rho}|u|^{2}\right\|_{2}^{2}-\int(\mu \Delta u & +(\mu+\lambda) \nabla \operatorname{div} u) \cdot|u|^{2} u d x=-\int p \operatorname{div}\left(|u|^{2} u\right) d x \\
& \leq\left(\mu-\frac{\lambda}{2}\right) \int|u|^{2}|\nabla u|^{2} d x+C \int \rho^{2} \theta^{2}|u|^{2} d x
\end{aligned}
$$

Some elementary calculations show that

$$
-\int(\mu \Delta u+(\mu+\lambda) \nabla \operatorname{div} u) \cdot|u|^{2} u d x \geq(2 \mu-\lambda) \int|u|^{2}|\nabla u|^{2} d x .
$$

Combining the above two inequalities leads to

$$
\frac{d}{d t}\left\|\sqrt{\rho}|u|^{2}\right\|_{2}^{2}+2(2 \mu-\lambda)\||u| \nabla u\|_{2}^{3} \lesssim \int \rho^{2} \theta^{2}|u|^{2} d x
$$

Multiplying (2.3) by a sufficient large number $K$ depending only on $R, \gamma, \mu, \lambda$, and $\kappa$, and summing the resultant with (2.2), one obtains

$$
\frac{d}{d t}\left(\|\sqrt{\rho} E\|_{2}^{2}+K\left\|\sqrt{\rho}|u|^{2}\right\|_{2}^{2}\right)+\kappa c_{v}\|\nabla \theta\|_{2}^{2}+(2 \mu-\lambda) K\||u| \nabla u\|_{2}^{2} \lesssim \int \rho^{2} \theta^{2}|u|^{2} d x
$$

from which, noticing that the Hölder and Sobolev inequalities yield

$$
\begin{aligned}
\int \rho^{2} \theta^{2}|u|^{2} d x & \leq\|\sqrt{\rho} \theta\|_{2}\|\theta\|_{6}\left\||u|^{2}\right\|_{6}\|\rho\|_{9}^{\frac{3}{2}} \\
& \lesssim\|\sqrt{\rho} \theta\|_{2}\|\nabla \theta\|_{2}\left\|\nabla|u|^{2}\right\|_{2}\|\rho\|_{\infty}\|\rho\|_{3}^{\frac{1}{2}},
\end{aligned}
$$

one obtains

$$
\begin{aligned}
\frac{d}{d t}\left(\|\sqrt{\rho} E\|_{2}^{2}+K\left\|\sqrt{\rho}|u|^{2}\right\|_{2}^{2}\right) & +\kappa c_{v}\|\nabla \theta\|_{2}^{2}+(2 \mu-\lambda) K\||u| \nabla u\|_{2}^{2} \\
& \lesssim\|\rho\|_{\infty}\|\rho\|_{3}^{\frac{1}{2}}\|\sqrt{\rho} \theta\|_{2}\|\nabla \theta\|_{2}\left\|\nabla|u|^{2}\right\|_{2} .
\end{aligned}
$$

Integrating this in $t$ and using the Cauchy inequality, the conclusion follows.

The following proposition on the $L^{\infty}\left(0, T ; L^{3}\right)$ estimate for $\rho$ is crucial in the proof of this paper.

Proposition 2.4. The following estimate holds

$$
\begin{aligned}
\sup _{0 \leq t \leq T}\|\rho\|_{3}^{3}+\int_{0}^{T} \int \rho^{3} p d x d t \leq & C \sup _{0 \leq t \leq T}\left(\|\rho\|_{\infty}^{\frac{2}{3}}\|\sqrt{\rho} u\|_{2}^{\frac{1}{3}}\left\|\sqrt{\rho}|u|^{2}\right\|_{2}^{\frac{1}{3}}\|\rho\|_{3}^{3}\right) \\
& +C \int_{0}^{T}\|\rho\|_{\infty}^{2}\|\rho\|_{3}^{2}\|\nabla u\|_{2}^{2} d t+C\left\|\rho_{0}\right\|_{3}^{3},
\end{aligned}
$$

for a positive constant $C$ depending only on $R, \gamma, \mu, \lambda$, and $\kappa$. 
Proof. Applying the operator $\Delta^{-1}$ div to (1.2) yields

$$
\Delta^{-1} \operatorname{div}(\rho u)_{t}+\Delta^{-1} \operatorname{div} \operatorname{div}(\rho u \otimes u)-(2 \mu+\lambda) \operatorname{div} u+p=0 .
$$

Multiplying the above equation by $\rho^{3}$ and noticing that

$$
\partial_{t} \rho^{3}+\operatorname{div}\left(u \rho^{3}\right)+2 \operatorname{div} u \rho^{3}=0,
$$

one obtains

$$
\frac{2 \mu+\lambda}{2}\left(\partial_{t} \rho^{3}+\operatorname{div}\left(u \rho^{3}\right)\right)+\rho^{3} p+\rho^{3} \Delta^{-1} \operatorname{div}(\rho u)_{t}+\rho^{3} \Delta^{-1} \operatorname{div} \operatorname{div}(\rho u \otimes u)=0 .
$$

Integrating the above equation over $\mathbb{R}^{3}$ yields

$$
\begin{array}{r}
\frac{2 \mu+\lambda}{2} \frac{d}{d t}\|\rho\|_{3}^{3}+\int \rho^{3} p d x+\int \rho^{3} \Delta^{-1} \operatorname{div}(\rho u)_{t} d x \\
=-\int \rho^{3} \Delta^{-1} \operatorname{div} \operatorname{div}(\rho u \otimes u) d x
\end{array}
$$

Using (1.1), one deduces

$$
\begin{aligned}
& \int \rho^{3} \Delta^{-1} \operatorname{div}(\rho u)_{t} d x \\
= & \frac{d}{d t} \int \rho^{3} \Delta^{-1} \operatorname{div}(\rho u) d x+\int\left[\operatorname{div}\left(\rho^{3} u\right)+2 \operatorname{div} u \rho^{3}\right] \Delta^{-1} \operatorname{div}(\rho u) d x \\
= & \int\left[2 \operatorname{div} u \rho^{3} \Delta^{-1} \operatorname{div}(\rho u)-\rho^{3} u \cdot \nabla \Delta^{-1} \operatorname{div}(\rho u)\right] d x \\
& +\frac{d}{d t} \int \rho^{3} \Delta^{-1} \operatorname{div}(\rho u) d x .
\end{aligned}
$$

Therefore, it follows from (2.7) that

$$
\begin{aligned}
& \frac{d}{d t} \int\left(\frac{2 \mu+\lambda}{2}+\Delta^{-1} \operatorname{div}(\rho u)\right) \rho^{3} d x+\int \rho^{3} p d x \\
= & \int\left[\rho^{3}\left(u \cdot \nabla \Delta^{-1} \operatorname{div}(\rho u)-\Delta^{-1} \operatorname{divdiv}(\rho u \otimes u)\right)-2 \operatorname{div} u \rho^{3} \Delta^{-1} \operatorname{div}(\rho u)\right] d x .
\end{aligned}
$$

Noticing that

$$
\begin{aligned}
\left\|\nabla \Delta^{-1} \operatorname{div}(\rho u)\right\|_{2} & \lesssim\|\rho u\|_{2} \lesssim\|\rho\|_{3}\|u\|_{6}, \\
\left\|\Delta^{-1} \operatorname{div} \operatorname{div}(\rho u \otimes u)\right\|_{\frac{3}{2}} & \lesssim\left\|\rho|u|^{2}\right\|_{\frac{3}{2}} \lesssim\|\rho\|_{3}\|u\|_{6}^{2},
\end{aligned}
$$

it follows from the Hölder and Sobolev embedding inequality that

$$
\begin{array}{r}
\int \rho^{3}\left(u \cdot \nabla \Delta^{-1} \operatorname{div}(\rho u)-\Delta^{-1} \operatorname{div} \operatorname{div}(\rho u \otimes u)\right) d x \\
\lesssim\|\rho\|_{9}^{3}\|\rho\|_{3}\|u\|_{6}^{2} \lesssim\|\rho\|_{\infty}^{2}\|\rho\|_{3}^{2}\|\nabla u\|_{2}^{2} .
\end{array}
$$


By the Sobolev embedding and elliptic estimates

$$
\begin{aligned}
\left\|\Delta^{-1} \operatorname{div}(\rho u)\right\|_{6} & \lesssim\left\|\nabla \Delta^{-1} \operatorname{div}(\rho u)\right\|_{2} \lesssim\|\rho u\|_{2} \\
& \lesssim\|\rho\|_{3}\|u\|_{6} \lesssim\|\rho\|_{3}\|\nabla u\|_{2},
\end{aligned}
$$

and, thus, the Hölder inequality yields

$$
\left|\int \operatorname{div} u \rho^{3} \Delta^{-1} \operatorname{div}(\rho u) d x\right| \lesssim\|\operatorname{div} u\|_{2}\|\rho\|_{9}^{3}\|\rho\|_{3}\|\nabla u\|_{2} \lesssim\|\rho\|_{\infty}^{2}\|\rho\|_{3}^{2}\|\nabla u\|_{2}^{2}
$$

By the Gagliardo-Nirenberg inequality and using the elliptic estimates, it follows

$$
\begin{aligned}
\left\|\Delta^{-1} \operatorname{div}(\rho u)\right\|_{\infty} & \lesssim\left\|\Delta^{-1} \operatorname{div}(\rho u)\right\|_{6}^{\frac{1}{3}}\left\|\nabla \Delta^{-1} \operatorname{div}(\rho u)\right\|_{4}^{\frac{2}{3}} \\
& \lesssim\|\rho u\|_{2}^{\frac{1}{3}}\|\rho u\|_{4}^{\frac{2}{3}} \lesssim\|\rho\|_{\infty}^{\frac{2}{3}}\|\sqrt{\rho} u\|_{2}^{\frac{1}{3}}\left\|\sqrt{\rho}|u|^{2}\right\|_{2}^{\frac{1}{3}} .
\end{aligned}
$$

Integrating (2.8) in $t$, using (2.9) $-(2.11)$, and by some straightforward calculations, the conclusion follows.

Proposition 2.5. Assume

$$
\sup _{0 \leq t \leq T}\|\rho\|_{\infty} \leq 4 \bar{\rho}
$$

Then, there is a positive constant $C$ depending only on $R, \gamma, \mu, \lambda$, and $\kappa$, such that

$$
\begin{aligned}
& \sup _{0 \leq t \leq T}\|\nabla u\|_{2}^{2}+\int_{0}^{T}\left\|\left(\sqrt{\rho} u_{t}, \frac{\nabla G}{\sqrt{\bar{\rho}}}, \frac{\nabla \omega}{\sqrt{\bar{\rho}}}\right)\right\|_{2}^{2} d t \\
\leq & C\left\|\nabla u_{0}\right\|_{2}^{2}+C \bar{\rho} \sup _{0 \leq t \leq T}\|\sqrt{\rho} \theta\|_{2}^{2}+C \bar{\rho}^{3} \int_{0}^{T}\|\nabla u\|_{2}^{4}\|(\nabla u, \sqrt{\bar{\rho}} \sqrt{\rho} \theta)\|_{2}^{2} d t \\
& +C \int_{0}^{T}\left(\bar{\rho}+\bar{\rho}^{2}\|\rho\|_{3}^{\frac{1}{2}}\|\sqrt{\rho} \theta\|_{2}\right)\|(\nabla \theta,|u| \nabla u)\|_{2}^{2} d t,
\end{aligned}
$$

where $G=(2 \mu+\lambda)$ divu $-p$ and $\omega=\nabla \times u$.

Proof. Multiplying (1.2) by $u_{t}$, integrating the resultant over $\mathbb{R}^{3}$, it follows from integration by parts that

$$
\begin{aligned}
\frac{1}{2} \frac{d}{d t}\left(\mu\|\nabla u\|_{2}^{2}+(\mu+\lambda)\|\operatorname{div} u\|_{2}^{2}\right)- & \int p \operatorname{div} u_{t} d x+\left\|\sqrt{\rho} u_{t}\right\|_{2}^{2} \\
& =-\int \rho(u \cdot \nabla) u \cdot u_{t} d x
\end{aligned}
$$

Noticing that $\operatorname{div} u=\frac{G+p}{2 \mu+\lambda}$, it follows

$$
\begin{gathered}
-\int p \operatorname{div} u_{t} d x=-\frac{d}{d t} \int p \operatorname{div} u d x+\int p_{t} \operatorname{div} u d x \\
=-\frac{d}{d t} \int p \operatorname{div} u d x+\frac{1}{2(2 \mu+\lambda)} \frac{d}{d t}\|p\|_{2}^{2}+\frac{1}{2 \mu+\lambda} \int p_{t} G d x .
\end{gathered}
$$


Noticing that (1.3) implies

$$
p_{t}=(\gamma-1)(\mathcal{Q}(\nabla u)-p \operatorname{div} u+\kappa \Delta \theta)-\operatorname{div}(u p),
$$

and, thus, integration by parts gives

$$
\int p_{t} G d x=\int[(\gamma-1)(\mathcal{Q}(\nabla u)-p \operatorname{div} u) G+(u p-\kappa(\gamma-1) \nabla \theta) \cdot \nabla G] d x .
$$

Substituting (2.14) into (2.13), then the resultant into (2.12), and noticing that $\|\nabla u\|_{2}^{2}=\|\omega\|_{2}^{2}+\|\operatorname{div} u\|_{2}^{2}$, by some straightforward calculations, one obtains

$$
\begin{aligned}
& \frac{1}{2} \frac{d}{d t}\left(\mu\|\omega\|_{2}^{2}+\frac{\|G\|_{2}^{2}}{2 \mu+\lambda}\right)+\left\|\sqrt{\rho} u_{t}\right\|_{2}^{2} \\
= & -\int \rho(u \cdot \nabla) u \cdot u_{t} d x+\frac{1}{2 \mu+\lambda} \int(\kappa(\gamma-1) \nabla \theta-u p) \cdot \nabla G d x \\
& -\frac{\gamma-1}{2 \mu+\lambda} \int(\mathcal{Q}(\nabla u)-p \operatorname{div} u) G d x .
\end{aligned}
$$

Use $\Delta u=\nabla \operatorname{div} u-\nabla \times \nabla \times u$ to rewrite (1.2) as

$$
\rho\left(u_{t}+u \cdot \nabla u\right)=\nabla G-\mu \nabla \times \omega .
$$

Testing this by $\nabla G$, noticing $\int \nabla G \cdot \nabla \times \omega d x=0$, and recalling $\|\rho\|_{\infty} \leq 4 \bar{\rho}$ yield

$$
\begin{aligned}
\|\nabla G\|_{2}^{2} & =\int \rho\left(u_{t}+u \cdot \nabla u\right) \cdot \nabla G d x \\
& \leq \int\left(\frac{|\nabla G|^{2}}{2}+2 \bar{\rho} \rho\left|u_{t}\right|^{2}\right) d x+\int \rho u \cdot \nabla u \cdot \nabla G d x
\end{aligned}
$$

which gives

$$
\frac{\|\nabla G\|_{2}^{2}}{16 \bar{\rho}} \leq \frac{1}{4}\left\|\sqrt{\rho} u_{t}\right\|_{2}^{2}+\frac{1}{8 \bar{\rho}} \int \rho(u \cdot \nabla) u \cdot \nabla G d x
$$

Similarly

$$
\frac{\mu^{2}\|\nabla \omega\|_{2}^{2}}{16 \bar{\rho}} \leq \frac{1}{4}\left\|\sqrt{\rho} u_{t}\right\|_{2}^{2}+\frac{1}{8 \bar{\rho}} \int \rho(u \cdot \nabla) u \cdot \nabla \times \omega d x .
$$

Thanks to (2.17) and (2.18), one obtains from (2.15) that

$$
\begin{aligned}
& \frac{1}{2} \frac{d}{d t}\left(\mu\|\omega\|_{2}^{2}+\frac{\|G\|_{2}^{2}}{2 \mu+\lambda}\right)+\frac{1}{2}\left\|\sqrt{\rho} u_{t}\right\|_{2}^{2}+\frac{1}{16 \bar{\rho}}\left(\|\nabla G\|_{2}^{2}+\mu^{2}\|\nabla \omega\|_{2}^{2}\right) \\
\leq & C \int \rho|u||\nabla u|\left[\left|u_{t}\right|+\frac{1}{\bar{\rho}}(\nabla G|+| \nabla \omega \mid)\right] d x+C \int(|\nabla \theta|+\rho \theta|u|)|\nabla G| d x \\
& +C \int\left(|\nabla u|^{2}+\rho \theta|\nabla u|\right)|G| d x=: I_{1}+I_{2}+I_{3} .
\end{aligned}
$$


The terms $I_{1}, I_{2}$, and $I_{3}$ are estimated as follows. For $I_{1}$, by the Hölder and Young inequalities, one obtains

$$
\begin{aligned}
I_{1} & \lesssim \sqrt{\bar{\rho}}\||u| \nabla u\|_{2}\left\|\sqrt{\rho} u_{t}\right\|_{2}+\||u| \nabla u\|_{2}\left(\|\nabla G\|_{2}+\|\nabla \omega\|_{2}\right) \\
& \leq \frac{1}{6}\left[\frac{1}{2}\left\|\sqrt{\rho} u_{t}\right\|_{2}^{2}+\frac{1}{16 \bar{\rho}}\left(\|\nabla G\|_{2}^{2}+\mu^{2}\|\nabla \omega\|_{2}^{2}\right)\right]+C \bar{\rho}\||u| \nabla u\|_{2}^{2} .
\end{aligned}
$$

Recalling (2.4), it follows from the Hölder and Young inequalities that

$$
\begin{aligned}
I_{2} & \lesssim\|\nabla \theta\|_{2}\|\nabla G\|_{2}+\|\rho \theta u\|_{2}\|\nabla G\|_{2} \\
& \lesssim\|\nabla \theta\|_{2}\|\nabla G\|_{2}+\sqrt{\bar{\rho}}\|\rho\|_{3}^{\frac{1}{4}}\|\sqrt{\rho} \theta\|_{2}^{\frac{1}{2}}\|\nabla \theta\|_{2}^{\frac{1}{2}}\left\|\nabla|u|^{2}\right\|_{2}^{\frac{1}{2}}\|\nabla G\|_{2} \\
& \leq \frac{\|\nabla G\|_{2}^{2}}{96 \bar{\rho}}+C \bar{\rho}^{2}\|\rho\|_{2}^{\frac{1}{2}}\|\sqrt{\rho} \theta\|_{2}\left(\|\nabla \theta\|_{2}^{2}+\||u| \nabla u\|_{2}^{2}\right) .
\end{aligned}
$$

The elliptic estimates and Sobolev embedding inequality yield

$$
\begin{aligned}
\|\nabla u\|_{6} & \lesssim\|\nabla \times u\|_{6}+\|\operatorname{div} u\|_{6} \lesssim\|\omega\|_{6}+\|G\|_{6}+\|\rho \theta\|_{6} \\
& \lesssim\|\nabla \omega\|_{2}+\|\nabla G\|_{2}+\bar{\rho}\|\nabla \theta\|_{2} .
\end{aligned}
$$

Using (2.20), by the Hölder, Sobolev, and Young inequalities, one deduces

$$
\begin{aligned}
I_{3} \lesssim & \|\nabla u\|_{2}\|\nabla u\|_{6}\|G\|_{3}+\|\nabla u\|_{2}\|\rho \theta\|_{6}\|G\|_{3} \\
\lesssim & C\|\nabla u\|_{2}\left(\|\nabla G\|_{2}+\|\nabla \omega\|_{2}+\bar{\rho}\|\nabla \theta\|_{2}\right)\|G\|_{2}^{\frac{1}{2}} \\
& +\|\nabla G\|_{2}^{\frac{1}{2}} \bar{\rho}\|\nabla u\|_{2}\|\nabla \theta\|_{2}\|G\|_{2}^{\frac{1}{2}}\|\nabla G\|_{2}^{\frac{1}{2}} \\
\leq & \frac{1}{96 \bar{\rho}}\left(\|\nabla G\|_{2}^{2}+\mu^{2}\|\nabla \omega\|_{2}^{2}\right)+C \bar{\rho}^{3}\|\nabla u\|_{2}^{4}\|G\|_{2}^{2} \\
& +C \bar{\rho}\left(\|\nabla \theta\|_{2}^{2}+\|u \mid \nabla u\|_{2}^{2}\right) .
\end{aligned}
$$

Substituting the estimates for $I_{i}, i=1,2,3$, into (2.19) yields

$$
\begin{aligned}
& \frac{d}{d t}\left(\mu\|\omega\|_{2}^{2}+\frac{\|G\|_{2}^{2}}{2 \mu+\lambda}\right)+\frac{1}{2}\left\|\sqrt{\rho} u_{t}\right\|_{2}^{2}+\frac{1}{16 \bar{\rho}}\left(\|\nabla G\|_{2}^{2}+\mu^{2}\|\nabla \omega\|_{2}^{2}\right) \\
& \quad \lesssim\left(\bar{\rho}+\bar{\rho}^{2}\|\rho\|_{3}^{\frac{1}{2}}\|\sqrt{\rho} \theta\|_{2}\right)\left(\|\nabla \theta\|_{2}^{2}+\||u| \nabla u\|_{2}^{2}\right)+\bar{\rho}^{3}\|\nabla u\|_{2}^{4}\|G\|_{2}^{2},
\end{aligned}
$$

from which, integrating in $t$ and using

$$
\|\nabla u\|_{2} \lesssim\|\omega\|_{2}+\|G\|_{2}+\|\rho \theta\|_{2} \lesssim\|\omega\|_{2}+\|G\|_{2}+\sqrt{\bar{\rho}}\|\sqrt{\rho} \theta\|_{2},
$$

the conclusion follows by straightforward calculations.

Proposition 2.6. Assume

$$
\sup _{0 \leq t \leq T}\|\rho\|_{\infty} \leq 4 \bar{\rho}
$$

Then, there is a positive constant $C$ depending only on $R, \gamma, \mu, \lambda$, and $\kappa$, such that

$$
\sup _{0 \leq t \leq T}\|\rho\|_{\infty} \leq\left\|\rho_{0}\right\|_{\infty} e^{C \bar{\rho}^{\frac{2}{3}} \sup _{0 \leq t \leq T}\|\sqrt{\rho} u\|_{2}^{\frac{1}{3}}\left\|\sqrt{\rho}|u|^{2}\right\|_{2}^{\frac{1}{3}}+C \bar{\rho} \int_{0}^{T}\|\nabla u\|_{2}\|(\nabla G, \nabla \omega, \sqrt{\bar{\rho}} \nabla \theta)\|_{2} d t} .
$$


Proof. Denote $\mathcal{O}=\left\{x \in \mathbb{R}^{3} \mid \rho_{0}(x)=0\right\}$ and $\Omega=\left\{x \in \mathbb{R}^{3} \mid \rho_{0}(x)>0\right\}$. Let $X(x, t)$ be the particle path starting from $x$ and govern by the velocity field $u$, that is

$$
\partial_{t} X(x, t)=u(X(x, t), t), \quad X(x, 0)=x .
$$

Then $\rho(X(x, t), t) \equiv 0$, for any $x \in \mathcal{O}$, and $\rho(X(x, t), t)>0$, for any $x \in \Omega$. One can verify that $\left\{X(x, t) \mid x \in \mathbb{R}^{3}\right\}=\mathbb{R}^{3}$, for any $t \in(0, T)$. Therefore

$$
\sup _{x \in \mathbb{R}^{3}} \rho(x, t)=\sup _{x \in \mathbb{R}^{3}}\|\rho(X(x, t), t)\|_{\infty}=\sup _{x \in \Omega} \rho(X(x, t), t) .
$$

Rewrite (2.5) as

$$
\begin{aligned}
& \partial_{t} \Delta^{-1} \operatorname{div}(\rho u)+u \cdot \nabla \Delta^{-1} \operatorname{div} \operatorname{div}(\rho u)-(2 \mu+\lambda) \operatorname{div} u+p \\
= & u \cdot \nabla \Delta^{-1} \operatorname{div} \operatorname{div}(\rho u)-\Delta^{-1} \operatorname{div} \operatorname{div}(\rho u \otimes u)=[u, \mathcal{R} \otimes \mathcal{R}](\rho u),
\end{aligned}
$$

where $\mathcal{R}$ is the Riesz transform on $\mathbb{R}^{3}$. Using the fact $\frac{d}{d t}(f(X(x, t), t))=\left(\partial_{t} f+u\right.$. $\nabla f)(X(x, t), t)$, it follows from (1.1) that

$$
\frac{d}{d t}(\log \rho(X(x, t), t))=-\operatorname{div} u(X(x, t), t), \quad \forall x \in \Omega .
$$

Therefore, for any $x \in \Omega$, it follows from (2.22) that

$$
\begin{array}{r}
\frac{d}{d t}\left((2 \mu+\lambda) \log \rho(X(x, t), t)+\left(\Delta^{-1} \operatorname{div}(\rho u)\right)(X(x, t), t)\right) \\
+p(X(x, t), t)=([u, \mathcal{R} \otimes \mathcal{R}](\rho u))(X(x, t), t) .
\end{array}
$$

Due to $p \geq 0$ and (2.21), one can easily derive from the above equality that

$$
\|\rho\|_{\infty} \leq\left\|\rho_{0}\right\|_{\infty} e^{C\left(\sup _{0 \leq t \leq T}\left\|\Delta^{-1} \operatorname{div}(\rho u)\right\|_{\infty}+\int_{0}^{T}\|[u, \mathcal{R} \otimes \mathcal{R}](\rho u)\|_{\infty} d t\right)} .
$$

Using the Gagliardo-Nirenberg inequality and the commutator estimates, one deduces

$$
\begin{aligned}
&\|[u, \mathcal{R} \otimes \mathcal{R}](\rho u)\|_{\infty} \lesssim\|[u, \mathcal{R} \otimes \mathcal{R}](\rho u)\|_{3}^{\frac{1}{5}}\|\nabla[u, \mathcal{R} \otimes \mathcal{R}](\rho u)\|_{4}^{\frac{4}{5}} \\
& \lesssim\|u\|_{6}^{\frac{1}{5}}\|\rho u\|_{6}^{\frac{1}{5}}\|\nabla u\|_{6}^{\frac{4}{5}}\|\rho u\|_{12}^{\frac{4}{5}} \lesssim \bar{\rho}\|u\|_{6}^{\frac{1}{5}}\|u\|_{6}^{\frac{1}{5}}\|\nabla u\|_{6}^{\frac{4}{5}}\left(\|u\|_{6}^{\frac{3}{4}}\|\nabla u\|_{6}^{\frac{1}{4}}\right)^{\frac{4}{5}} \\
& \lesssim \bar{\rho}\|\nabla u\|_{2}\|\nabla u\|_{6} \lesssim \bar{\rho}\|\nabla u\|_{2}\left(\|\nabla G\|_{2}+\|\nabla \omega\|_{2}+\bar{\rho}\|\nabla \theta\|_{2}\right),
\end{aligned}
$$

where, in the last step, (2.20) has been used. Thanks to this and recalling (2.11), the conclusion follows from (2.23).

\subsection{A priori estimates.}

Proposition 2.7. Assume that $2 \mu>\lambda$. Denote

$$
\mathscr{N}_{T}=\bar{\rho} \sup _{0 \leq t \leq T}\left(\|\rho\|_{3}+\bar{\rho}^{2}\|\sqrt{\rho} u\|_{2}^{2}\right)(t) \sup _{0 \leq t \leq T}\left(\|\nabla u\|_{2}^{2}+\bar{\rho}\|\sqrt{\rho} E\|_{2}^{2}\right)(t) .
$$

Then, there is a positive constant $\eta_{0}$ depending only on $R, \gamma, \mu, \lambda$, and $\kappa$, such that if

$$
\eta \leq \eta_{0}, \quad \sup _{0 \leq t \leq T}\|\rho\|_{\infty} \leq 4 \bar{\rho}, \quad \text { and } \quad \mathscr{N}_{T} \leq \sqrt{\eta}
$$



then the following estimates hold

$$
\begin{aligned}
\sup _{0 \leq t \leq T}\|\sqrt{\rho} E\|_{2}^{2}+\int_{0}^{T}\|(\nabla \theta,|u| \nabla u)\|_{2}^{2} d t & \leq C\left\|\sqrt{\rho_{0}} E_{0}\right\|_{2}^{2}, \\
\sup _{0 \leq t \leq T}\|\rho\|_{3}+\left(\int_{0}^{T} \int \rho^{3} p d x d t\right)^{\frac{1}{3}} & \leq C\left(\left\|\rho_{0}\right\|_{3}+\bar{\rho}^{2}\left\|\sqrt{\rho_{0}} u_{0}\right\|_{2}^{2}\right), \\
\bar{\rho}^{2}\left(\sup _{0 \leq t \leq T}\|\sqrt{\rho} u\|_{2}^{2}+\int_{0}^{T}\|\nabla u\|_{2}^{2} d t\right) & \leq C\left(\left\|\rho_{0}\right\|_{3}+\bar{\rho}^{2}\left\|\sqrt{\rho_{0}} u_{0}\right\|_{2}^{2}\right), \\
\sup _{0 \leq t \leq T}\|\nabla u\|_{2}^{2}+\int_{0}^{T}\left\|\left(\sqrt{\rho} u_{t}, \frac{\nabla G}{\sqrt{\bar{\rho}}}, \frac{\nabla \omega}{\sqrt{\bar{\rho}}}\right)\right\|_{2}^{2} d t & \leq C\left(\left\|\nabla u_{0}\right\|_{2}^{2}+\bar{\rho}\left\|\sqrt{\rho_{0}} E_{0}\right\|_{2}^{2}\right), \\
\sup _{0 \leq t \leq T}\|\rho\|_{\infty} & \leq \bar{\rho} e^{C \mathscr{N}_{0}^{\frac{1}{6}}+C \mathscr{N}_{0}^{\frac{1}{2}}},
\end{aligned}
$$

for a positive constant $C$ depending only on $R, \gamma, \mu, \lambda$, and $\kappa$, where

$$
\mathscr{N}_{0}=\bar{\rho}\left(\left\|\rho_{0}\right\|_{3}+\bar{\rho}^{2}\left\|\sqrt{\rho_{0}} u_{0}\right\|_{2}^{2}\right)\left(\left\|\nabla u_{0}\right\|_{2}^{2}+\bar{\rho}\left\|\sqrt{\rho_{0}} E_{0}\right\|_{2}^{2}\right) \text {. }
$$

Proof. By assumptions, it follows from Proposition 2.3 that

$$
\begin{gathered}
\sup _{0 \leq t \leq T}\|\sqrt{\rho} E\|_{2}^{2}+\int_{0}^{T}\left(\|\nabla \theta\|_{2}^{2}+\||u| \nabla u\|_{2}^{2}\right) d t \\
\leq C\left\|\sqrt{\rho_{0}} E_{0}\right\|_{2}^{2}+C \eta_{0}^{\frac{1}{4}} \int_{0}^{T}\left(\|\nabla \theta\|_{2}^{2}+\||u| \nabla u\|_{2}^{2}\right) d t
\end{gathered}
$$

which by choosing $\eta_{0}$ suitably small implies

$$
\sup _{0 \leq t \leq T}\|\sqrt{\rho} E\|_{2}^{2}+\int_{0}^{T}\left(\|\nabla \theta\|_{2}^{2}+\||u| \nabla u\|_{2}^{2}\right) d t \leq C\left\|\sqrt{\rho_{0}} E_{0}\right\|_{2}^{2} .
$$

Thanks to (2.24) and applying Proposition 2.2, one obtains

$$
\begin{aligned}
\sup _{0 \leq t \leq T}\|\sqrt{\rho} u\|_{2}^{2}+\int_{0}^{T}\|\nabla u\|_{2}^{2} d t & \leq C\left\|\sqrt{\rho_{0}} u_{0}\right\|_{2}^{2}+C\left\|\sqrt{\rho_{0}} E_{0}\right\|_{2}^{2} \sup _{0 \leq t \leq T}\|\rho\|_{3}^{2} \\
& \leq C\left\|\sqrt{\rho_{0}} u_{0}\right\|_{2}^{2}+C \sup _{0 \leq t \leq T}\|\sqrt{\rho} E\|_{2}^{2} \sup _{0 \leq t \leq T}\|\rho\|_{3}^{2} \\
& \leq C\left\|\sqrt{\rho_{0}} u_{0}\right\|_{2}^{2}+\frac{C \sqrt{\eta_{0}}}{\bar{\rho}^{2}} \sup _{0 \leq t \leq T}\|\rho\|_{3} .
\end{aligned}
$$

Using the assumptions and (2.25), it follows from Proposition 2.4 and the Young inequality that

$$
\sup _{0 \leq t \leq T}\|\rho\|_{3}^{3}+\int_{0}^{T} \int \rho^{3} p d x d t
$$




$$
\begin{aligned}
& \leq C\left\|\rho_{0}\right\|_{3}^{3}+C \eta_{0}^{\frac{1}{12}} \sup _{0 \leq t \leq T}\left\|\rho_{0}\right\|_{3}^{3}+C \bar{\rho}^{2}\left(\left\|\sqrt{\rho_{0}} u_{0}\right\|_{2}^{2}+\frac{\sqrt{\eta_{0}}}{\bar{\rho}^{2}} \sup _{0 \leq t \leq T}\|\rho\|_{3}\right) \sup _{0 \leq t \leq T}\|\rho\|_{3}^{2} \\
& \leq C\left\|\rho_{0}\right\|_{3}^{3}+\left(C \eta_{0}^{\frac{1}{12}}+\frac{1}{4}+C \sqrt{\eta_{0}}\right) \sup _{0 \leq t \leq T}\|\rho\|_{3}^{3}+C \bar{\rho}^{6}\left\|\sqrt{\rho_{0}} u_{0}\right\|_{2}^{6},
\end{aligned}
$$

from which, by choosing $\eta_{0}$ sufficiently small, one obtains

$$
\sup _{0 \leq t \leq T}\|\rho\|_{3}+\left(\int_{0}^{T} \int \rho^{3} p d x d t\right)^{\frac{1}{3}} \leq C\left(\left\|\rho_{0}\right\|_{3}+\bar{\rho}^{2}\left\|\sqrt{\rho_{0}} u_{0}\right\|_{2}^{2}\right) .
$$

Combing (2.25) with (2.26) yields

$$
\bar{\rho}^{2}\left(\sup _{0 \leq t \leq T}\|\sqrt{\rho} u\|_{2}^{2}+\int_{0}^{T}\|\nabla u\|_{2}^{2} d t\right) \leq C\left(\left\|\rho_{0}\right\|_{3}+\bar{\rho}^{2}\left\|\sqrt{\rho_{0}} u_{0}\right\|_{2}^{2}\right) .
$$

Using (2.24) and (2.27), it follows from Proposition 2.5 that

$$
\begin{aligned}
& \sup _{0 \leq t \leq T}\|\nabla u\|_{2}^{2}+\int_{0}^{T}\left\|\left(\sqrt{\rho} u_{t}, \frac{\nabla G}{\sqrt{\bar{\rho}}}, \frac{\nabla \omega}{\sqrt{\bar{\rho}}}\right)\right\|_{2}^{2} d t \\
& \lesssim\left\|\nabla u_{0}\right\|_{2}^{2}+\bar{\rho}\left\|\sqrt{\rho_{0}} E_{0}\right\|_{2}^{2}+\bar{\rho}^{3} \int_{0}^{T}\|\nabla u\|_{2}^{2} d t \sup _{0 \leq t \leq T}\left(\|\nabla u\|_{2}^{2}+\bar{\rho}\|\sqrt{\rho} \theta\|_{2}^{2}\right) \\
& \times \sup _{0 \leq t \leq T}\|\nabla u\|_{2}^{2}+\left(\bar{\rho}+\bar{\rho}^{2} \sup _{0 \leq t \leq T}\|\rho\|_{3}^{\frac{1}{2}}\|\sqrt{\rho} \theta\|_{2}\right) \int_{0}^{T}\|(\nabla \theta,|u| \nabla u)\|_{2}^{2} d t \\
& \lesssim\left\|\nabla u_{0}\right\|_{2}^{2}+\bar{\rho}\left\|\sqrt{\rho_{0}} E_{0}\right\|_{2}^{2}+\bar{\rho}\left(\left\|\rho_{0}\right\|_{3}+\bar{\rho}^{2}\left\|\sqrt{\rho_{0}} u_{0}\right\|_{2}^{2}\right) \\
& \times \sup _{0 \leq t \leq T}\left(\|\nabla u\|_{2}^{2}+\bar{\rho}\|\sqrt{\rho} E\|_{2}^{2}\right) \sup _{0 \leq t \leq T}\|\nabla u\|_{2}^{2} \\
& +\bar{\rho}^{2} \sup _{0 \leq t \leq T}\|\rho\|_{3}^{\frac{1}{2}}\|\sqrt{\rho} \theta\|_{2}\left\|\sqrt{\rho_{0}} E_{0}\right\|_{2}^{2} .
\end{aligned}
$$

Recalling the definition of $\mathscr{N}_{T}$ and the assumption that $\mathscr{N}_{T} \leq \sqrt{\eta_{0}}$, it is clear that

$$
\begin{aligned}
& \bar{\rho}\left(\left\|\rho_{0}\right\|_{3}+\bar{\rho}^{2}\left\|\sqrt{\rho_{0}} u_{0}\right\|_{2}^{2}\right) \sup _{0 \leq t \leq T}\left(\|\nabla u\|_{2}^{2}+\bar{\rho}\|\sqrt{\rho} E\|_{2}^{2}\right) \\
\leq & \bar{\rho} \sup _{0 \leq t \leq T}\left(\|\rho\|_{3}+\bar{\rho}^{2}\|\sqrt{\rho} u\|_{2}^{2}\right) \sup _{0 \leq t \leq T}\left(\|\nabla u\|_{2}^{2}+\bar{\rho}\|\sqrt{\rho} E\|_{2}^{2}\right) \leq \mathscr{N}_{T} \leq \sqrt{\eta_{0}}
\end{aligned}
$$

and

$$
\bar{\rho} \sup _{0 \leq t \leq T}\|\rho\|_{3}^{\frac{1}{2}}\|\sqrt{\rho} \theta\|_{2} \leq\left(\bar{\rho}^{2} \sup _{0 \leq t \leq T}\|\rho\|_{3} \sup _{0 \leq t \leq T}\|\sqrt{\rho} E\|_{2}^{2}\right)^{\frac{1}{2}} \leq \mathscr{N}_{T}^{\frac{1}{2}} \leq \eta_{0}^{\frac{1}{4}} .
$$

Thanks to the above two estimates, by choosing $\eta_{0}$ sufficiently small, one can easily derive from (2.28) that

$$
\sup _{0 \leq t \leq T}\|\nabla u\|_{2}^{2}+\int_{0}^{T}\left\|\left(\sqrt{\rho} u_{t}, \frac{\nabla G}{\sqrt{\bar{\rho}}}, \frac{\nabla \omega}{\sqrt{\bar{\rho}}}\right)\right\|_{2}^{2} d t \leq C\left(\left\|\nabla u_{0}\right\|_{2}^{2}+\bar{\rho}\left\|\sqrt{\rho_{0}} E_{0}\right\|_{2}^{2}\right) .
$$


The estimate for $\|\rho\|_{\infty}$ follows from Proposition [2.6 by using (2.24), (2.27), and (2.29).

Proposition 2.8. Assume that $2 \mu>\lambda$. Let $\eta_{0}, \mathscr{N}_{T}$, and $\mathscr{N}_{0}$ be as in Proposition 2.7. Then, the following two hold:

(i) There is a number $\varepsilon_{0} \in\left(0, \eta_{0}\right)$ depending only on $R, \gamma, \mu, \lambda$, and $\kappa$, such that if

$$
\sup _{0 \leq t \leq T}\|\rho\|_{\infty} \leq 4 \bar{\rho}, \quad \mathscr{N}_{T} \leq \sqrt{\varepsilon_{0}}, \quad \text { and } \quad \mathscr{N}_{0} \leq \varepsilon_{0}
$$

then

$$
\sup _{0 \leq t \leq T}\|\rho\|_{\infty} \leq 2 \bar{\rho} \quad \text { and } \quad \mathscr{N}_{T} \leq \frac{\sqrt{\varepsilon_{0}}}{2} .
$$

(ii) As a consequence of (i), the following estimates hold

$$
\mathscr{N}_{T} \leq \frac{\sqrt{\varepsilon_{0}}}{2} \quad \text { and } \sup _{0 \leq t \leq T}\|\rho\|_{\infty} \leq 2 \bar{\rho}
$$

as long as $\mathscr{N}_{0} \leq \varepsilon_{0}$.

Proof. (i) Let $\varepsilon_{0} \leq \eta_{0}$ be sufficiently small. By assumptions, all the conditions in Proposition 2.7 hold, and, thus

$$
\begin{aligned}
\mathscr{N}_{T} & \leq C \bar{\rho}\left(\left\|\rho_{0}\right\|_{3}+\bar{\rho}^{2}\left\|\sqrt{\rho_{0}} u_{0}\right\|_{2}^{2}\right)\left(\left\|\nabla u_{0}\right\|_{2}^{2}+\bar{\rho}\left\|\sqrt{\rho_{0}} E_{0}\right\|_{2}^{2}\right) \\
& =C \mathscr{N}_{0} \leq C \varepsilon_{0} \leq \frac{\sqrt{\varepsilon_{0}}}{2}
\end{aligned}
$$

and

$$
\sup _{0 \leq t \leq T}\|\rho\|_{\infty} \leq \bar{\rho} e^{C \mathscr{N}_{0}^{\frac{1}{6}}+C \mathscr{N}_{0}^{\frac{1}{2}}} \leq \bar{\rho} e^{C \varepsilon_{0}^{\frac{1}{6}}+C \varepsilon_{0}^{\frac{1}{2}}} \leq 2 \bar{\rho}
$$

as long as $\varepsilon_{0}$ is sufficiently small. The first conclusion follows.

(ii) Define

$$
T_{\#}:=\max \left\{\mathcal{T} \in(0, T] \mid \mathscr{N}_{\mathcal{T}} \leq \sqrt{\varepsilon_{0}}, \sup _{0 \leq t \leq \mathcal{T}}\|\rho\|_{\infty} \leq 4 \bar{\rho}\right\} .
$$

Then, by (i), we have

$$
\mathscr{N}_{\mathcal{T}} \leq \frac{\sqrt{\varepsilon_{0}}}{2}, \quad \sup _{0 \leq t \leq \mathcal{T}}\|\rho\|_{\infty} \leq 2 \bar{\rho}, \quad \forall \mathcal{T} \in\left(0, T_{\#}\right) .
$$

If $T_{\#}<T$, noticing that $\mathscr{N}_{\mathcal{T}}$ and $\sup _{0 \leq t \leq \mathcal{T}}\|\rho\|_{\infty}$ are continuous on $[0, T]$, there is another time $T_{\# \#} \in\left(T_{\#}, T\right]$, such that

$$
\mathscr{N}_{T_{\# \#}} \leq \sqrt{\varepsilon_{0}} \quad \text { and } \quad \sup _{0 \leq t \leq T_{\# \#}}\|\rho\|_{\infty} \leq 4 \bar{\rho}
$$

which contradicts to the definition of $T_{\#}$. Thus, we have $T_{\#}=T$, and the conclusion follows from (2.30) and the continuity of $\mathscr{N}_{\mathcal{T}}$ and $\sup _{0 \leq t \leq \mathcal{T}}\|\rho\|_{\infty}$ on $[0, T]$. 
The following corollary is a straightforward consequence of Proposition 2.7 and (ii) of Proposition 2.8,

Corollary 2.1. Assume that $2 \mu>\lambda$. Let $\varepsilon_{0}$ be as in Proposition 2.8 and assume $\mathscr{N}_{0} \leq \varepsilon_{0}$. Then, there is a positive constant $C$ depending only on $R, \gamma, \mu, \lambda, \kappa, \bar{\rho}$, $\left\|\rho_{0}\right\|_{3},\left\|\sqrt{\rho_{0}} u_{0}\right\|_{2},\left\|\sqrt{\rho_{0}} E_{0}\right\|_{2}$, and $\left\|\nabla u_{0}\right\|_{2}$, such that the following estimates hold:

$$
\begin{array}{r}
\sup _{0 \leq t \leq T}\left(\|(\sqrt{\rho} E, \sqrt{\rho} u, \nabla u)\|_{2}^{2}+\|\rho\|_{3}+\|\rho\|_{\infty}\right) \leq C, \\
\int_{0}^{T}\left(\left\|\left(\nabla \theta,|u| \nabla u, \sqrt{\rho} u_{t}, \nabla G, \nabla \omega\right)\right\|_{2}^{2}+\|\nabla u\|_{6}^{2}+\int \rho^{3} p d x\right) d t \leq C .
\end{array}
$$

\section{Proof of Theorem 1.1}

The following blow-up criteria is cited from Huang-Li [20].

Proposition 3.1. Let $T^{*}<\infty$ be the maximal time of existence of a solution $(\rho, u, \theta)$ to system (1.1)-(1.3), with initial data $\left(\rho_{0}, u_{0}, \theta_{0}\right)$. Then,

$$
\lim _{T \rightarrow T^{*}}\left(\|\rho\|_{L^{\infty}\left(0, T ; L^{\infty}\right)}+\|u\|_{L^{s}\left(0, T ; L^{r}\right)}\right)=\infty
$$

for any $(s, r)$ such that $\frac{2}{s}+\frac{3}{r} \leq 1$ and $3<r \leq \infty$.

We are now ready to prove Theorem 1.1.

Proof of Theorem 1.1. Let $\varepsilon_{0}$ and $\mathscr{N}_{T}$ be as in Proposition 2.8 and assume $\mathscr{N}_{0} \leq$ $\varepsilon_{0}$. By Proposition 2.1, there is a unique local strong solution $(\rho, u, \theta)$ to system (1.1) -(1.3), with initial data $\left(\rho_{0}, u_{0}, \theta_{0}\right)$. Extend the local solution $(\rho, u, \theta)$ to the maximal time of existence $T_{\max }$. If $T_{\max }=\infty$, then $(\rho, u, \theta)$ is a global solution and we are down. Assume that $T_{\max }<\infty$. Then, by the blow up criteria in Proposition 3.1, it holds

$$
\lim _{T \rightarrow T_{\max }}\left(\|\rho\|_{L^{\infty}\left(0, T ; L^{\infty}\right)}+\|u\|_{L^{4}\left(0, T ; L^{6}\right)}\right)=\infty .
$$

By Corollary 2.1, it follows $\sup _{0 \leq t \leq T}\left(\|\rho\|_{\infty}+\|\nabla u\|_{2}^{2}\right) \leq C$ which, by the Sobolev embedding inequality, gives

$$
\|\rho\|_{L^{\infty}\left(0, T ; L^{\infty}\right)}+\|u\|_{L^{4}\left(0, T ; L^{6}\right)} \leq C
$$

for any $T \in\left(0, T_{\max }\right)$, and for a positive constant $C$ independent of $T$. This implies

$$
\lim _{T \rightarrow T_{\max }}\left(\|\rho\|_{L^{\infty}\left(0, T ; L^{\infty}\right)}+\|u\|_{L^{4}\left(0, T ; L^{6}\right)}\right) \leq C<\infty,
$$

contradicting to (3.1). Therefore, we must have $T_{\max }=\infty$, proving Theorem 1.1.

\section{ACKNOWLEDGMENTS}

J.Li was partly supported by start-up fund 550-8S0315 of the South China Normal University, the NSFC under 11771156 and 11871005, and the Hong Kong RGC Grant CUHK-14302917. 


\section{REFERENCES}

[1] Bresch, D.; Jabin, P.-E: Global existence of weak solutions for compressible Navier-Stokes equations: thermodynamically unstable pressure and anisotropic viscous stress tensor, Ann. Math., (2) 188 (2018), no. 2, 577-684.

[2] Chen, G.-Q.; Hoff, D.; Trivisa, K.: Global solutions of the compressible NavierStokes equations with large discontinuous initial data, Comm. Partial Differential Equations, 25 (2000), 2233-2257.

[3] Chen, Q.; Miao, C.; Zhang, Z.: Global well-posedness for compressible NavierStokes equations with highly oscillating initial velocity, Communications on Pure and Applied Mathematics, 63 (2010), 1173-1224.

[4] Chen, Q.; Tan, Z.; Wang, Y.: Strong solutions to the incompressible magnetohydrodynamic equations, Math. Methods Appl. Sci., 34 (2011), 94-107.

[5] Choe, H. J.; Kim, H.: Strong solutions of the Navier-Stokes equations for nonhomogeneous incompressible fluids, Comm. Partial Differential Equations, 28 (2003), 1183-1201.

[6] Craig, W.; Huang, X.; Wang, Y.: Global wellposedness for the 3D inhomogeneous incompressible Navier-Stokes equations, J. Math. Fluid Mech., 15 (2013), 747758.

[7] Chikami, N.; Danchin, R.: On the well-posedness of the full compressible NaiverStokes system in critical Besov spaces, J. Differential Equations, 258 (2015), 3435-3467.

[8] Cho, Y.; Choe, H. J.; Kim, H.: Unique solvability of the initial boundary value problems for compressible viscous fluids, J. Math. Pures Appl., 83 (2004), 243275.

[9] Cho, Y.; Kim, H.: On classical solutions of the compressible Navier-Stokes equations with nonnegative initial densities, Manuscripta Math., 120 (2006), 91-129.

[10] Cho, Y.; Kim, H.: Existence results for viscous polytropic fluids with vacuum, J. Differential Equations, 228 (2006), 377-411.

[11] Danchin, R.: Global existence in critical spaces for flows of compressible viscous and heat-conductive gases, Arch. Ration. Mech. Anal., 160 (2001), 1-39.

[12] Danchin, R.; Xu, J.: Optimal decay estimates in the critical Lp framework for flows of compressible viscous and heat-conductive gases, J. Math. Fluid Mech., 20 (2018), no. 4, 1641-1665.

[13] Deckelnick, K.: Decay estimates for the compressible Navier-Stokes equations in unbounded domains, Math. Z., 209 (1992), 115-130.

[14] Fang, D.; Zhang, T.; Zi, R.: Global solutions to the isentropic compressible Navier-Stokes equations with a class of large initial data, SIAM J. Math. Anal., 50 (2018), no. 5, 4983-5026.

[15] Feireisl, E.; Novotný, A.; Petzeltová, H.: On the existence of globally defined weak solutions to the Navier-Stokes equations, J. Math. Fluid Mech., 3 (2001), 358-392. 
[16] Feireisl, E.: On the motion of a viscous, compressible, and heat conducting fluid, Indiana Univ. Math. J., 53 (2004), 1705-1738.

[17] Feireisl, E.: Dynamics of viscous compressible fluids, Oxford Lecture Series in Mathematics and its Applications, 26. Oxford University Press, Oxford, 2004. xii+212 pp.

[18] Graffi, D.: Il teorema di unicitá nella dinamica dei fluidi compressibili (Italian), J. Rational Mech. Anal., 2 (1953), 99-106.

[19] Hoff, D.: Discontinuous solutions of the Navier-Stokes equations for multidimensional flows of heat-conducting fluids, Arch. Rational Mech. Anal., 139 (1997), 303-354.

[20] Huang, X.; Li, J.: Serrin-type blowup criterion for viscous, compressible, and heat conducting Navier-Stokes and magnetohydrodynamic flows, Comm. Math. Phys., 324 (2013), no. 1, 147-171.

[21] Huang, X.; Li, J.: Global classical and weak solutions to the three-dimensional full compressible Navier-Stokes system with vacuum and large oscillations, Arch. Ration. Mech. Anal., 227 (2018), no. 3, 995-1059.

[22] Huang, X.; Li, J.; Xin, Z.: Global well-posedness of classical solutions with large oscillations and vacuum to the three-dimensional isentropic compressible NavierStokes equations, Comm. Pure Appl. Math., 65 (2012), 549-585.

[23] Huang, X.; Wang, Y.: Global strong solution of 3D inhomogeneous Navier-Stokes equations with density-dependent viscosity, J. Differential Equations, 259 (2015), 1606-1627.

[24] Itaya, N.: On the Cauchy problem for the system of fundamental equations describing the movement of compressible viscous fluids, Kodai Math. Sem. Rep., 23 (1971), 60-120.

[25] Jiang, S.: Global spherically symmetric solutions to the equations of a viscous polytropic ideal gas in an exterior domain, Commun. Math. Phys., 178 (1996), 339-374.

[26] Jiang, S.; Zhang, P.: Axisymmetric solutions of the 3D Navier-Stokes equations for compressible isentropic fluids, J. Math. Pures Appl., 82 (2003), 949-973.

[27] Jiang, S.; Zlotnik, A.: Global well-posedness of the Cauchy problem for the equations of a one-dimensional viscous heat-conducting gas with Lebesgue initial data, Proc. Roy. Soc. Edinburgh Sect. A, 134 (2004), 939-960.

[28] Kanel', Ja. I.: A model system of equations for the one-dimensional motion of a gas, (Russian) Differencial'nye Uravnenija, 4 1968, 721-734.

[29] Kazhikhov, A. V.: Cauchy problem for viscous gas equations, Siberian Math. J., 23 (1982), 44-49.

[30] Kazhikhov, A. V.; Shelukhin, V. V.: Unique global solution with respect to time of initial boundary value problems for one-dimensional equations of a viscous gas, J. Appl. Math. Mech., 41 (1977), 273-282.

[31] Kobayashi, T.; Shibata, Y.: Decay estimates of solutions for the equations of motion of compressible viscous and heat-conductive gases in an exterior domain 
in $\mathbb{R}^{3}$, Commun. Math. Phys., 200 (1999), 621-659.

[32] Li, H.; Wang, Y.; Xin, Z.: Non-existence of classical solutions with finite energy to the Cauchy problem of the compressible Navier-Stokes equations, Arch. Rational Mech. Anal., 232 (2019), 557-590

[33] Li, J.: Global strong solutions to the inhomogeneous incompressible nematic liquid crystal flow, Methods Appl. Anal., 22 (2015), 201-220.

[34] Li, J.: Local existence and uniqueness of strong solutions to the Navier-Stokes equations with nonnegative density, J. Differential Equations, 263 (2017), no. 10, 6512-6536.

[35] Li, J.: Global well-posedness of the 1D compressible Navier-Stokes equations with constant heat conductivity and nonnegative density, arXiv:1801.09395

[36] Li, J.; Liang, Z.: Some uniform estimates and large-time behavior of solutions to one-dimensional compressible Navier-Stokes system in unbounded domains with large data, Arch. Rational Mech. Anal., 220 (2016), 1195-1208.

[37] Li, J.; Xin, Z.: Global well-posedness and large time asymptotic Bbehavior of classical solutions to the compressible Navier-Stokes equations with vacuum, Ann. PDE (2019) 5: 7.

[38] Li, J.; Xin, Z.: Entropy-bounded solutions to the compressible Navier-Stokes equations: with far field vacuum, arXiv:1710.06571

[39] Li, J.; Xin, Z.: Entropy-bounded solutions to the heat conductive compressible Navier-Stokes equations, preprint.

[40] Li, J.; Xin, Z.: Entropy-bounded solutions to the multi-dimensional heat conductive compressible Navier-Stokes equations, in preparation.

[41] Lions, P. L.: Existence globale de solutions pour les équations de Navier-Stokes compressibles isentropiques, C. R. Acad. Sci. Paris Sér. I Math., 316 (1993), $1335-1340$.

[42] Lions, P. L.: Mathematical Topics in Fluid Mechanics, Vol. 2, Clarendon, Oxford, 1998.

[43] Lukaszewicz, G.: An existence theorem for compressible viscous and heat conducting fluids, Math. Methods Appl. Sci., 6 (1984), 234-247.

[44] Matsumura, A.; Nishida, T.: The initial value problem for the equations of motion of viscous and heat-conductive gases, J. Math. Kyoto Univ., 20 (1980), 67-104.

[45] Matsumura, A.; Nishida, T.: The initial boundary value problem for the equations of motion of compressible viscous and heat-conductive fluid, Preprint University of Wisconsin, MRC Technical Summary Report no. 2237 (1981).

[46] Matsumura, A.; Nishida, T.: Initial-boundary value problems for the equations of motion of general fluids, Computing methods in applied sciences and engineering, V (Versailles, 1981), 389-406, North-Holland, Amsterdam, 1982.

[47] Matsumura, A.; Nishida, T.: Initial boundary value problems for the equations of motion of compressible viscous and heat-conductive fluids, Commun. Math. Phys., 89 (1983), 445-464. 
[48] Mellet, A.; Vasseur, A.: A bound from below for the temperature in compressible Navier-Stokes equations, Monatsh Math, 157 (2009), 143-161.

[49] Nash, J.: Le problème de Cauchy pour les équations différentielles d'un fluide général, Bull. Soc. Math. Fr., 90 (1962), 487-497.

[50] Ponce, G.: Global existence of small solutions to a class of nonlinear evolution equations, Nonlinear Anal., 9 (1985), 399-418.

[51] Serrin, J.: On the uniqueness of compressible fluid motions, Arch. Rational Mech. Anal., 3 (1959), 271-288.

[52] Tani, A.: On the first initial-boundary value problem of compressible viscous fluid motion, Publ. Res. Inst. Math. Sci., 13 (1977), 193-253.

[53] Valli, A.: An existence theorem for compressible viscous fluids, Ann. Mat. Pura Appl., 130 (1982), 197-213; 132 (1982), 399-400.

[54] Valli, A.; Zajaczkowski, W. M.: Navier-Stokes equations for compressible fluids: global existence and qualitative properties of the solutions in the general case, Commun. Math. Phys., 103 (1986), 259-296.

[55] Vol'pert, A. I., Hudjaev, S. I.: On the Cauchy problem for composite systems of nonlinear differential equations, Math. USSR-Sb, 16 (1972), 517-544 [previously in Mat. Sb. (N.S.), 87 (1972), 504-528(in Russian)].

[56] Wen, H.; Ding, S.: Solutions of incompressible hydrodynamic flow of liquid crystals, Nonlinear Anal. Real World Appl., 12 (2011), 1510-1531.

[57] Zhang, J.: Global well-posedness for the incompressible Navier-Stokes equations with density-dependent viscosity coefficient, J. Differential Equations, 259 (2015), 1722-1742.

[58] Wen, H.; Zhu, C.: Global solutions to the three-dimensional full compressible Navier-Stokes equations with vacuum at infinity in some classes of large data, SIAM J. Math. Anal., 49 (2017), 162-221.

[59] Xin, Z.: Blowup of smooth solutions to the compressible Navier-Stokes equation with compact density, Comm. Pure Appl. Math., 51 (1998), 229-240.

[60] Xin, Z.; Yan, W.: On blowup of classical solutions to the compressible NavierStokes equations, Comm. Math. Phys., 321 (2013), 529-541.

[61] Zlotnik, A. A.; Amosov, A. A.: On stability of generalized solutions to the equations of one-dimensional motion of a viscous heat-conducting gas, Siberian Math. J., 38 (1997), 663-684.

[62] Zlotnik, A. A.; Amosov, A. A.: Stability of generalized solutions to equations of one-dimensional motion of viscous heat conducting gases, Math. Notes, 63 (1998), 736-746.

(Jinkai li) South China Research Center for Applied Mathematics and Interdisciplinary Studies, South China Normal University, Zhong Shan Avenue West 55, Tianhe District, Guangzhou 510631, China

E-mail address: jklimath@m.scnu.edu.cn; jklimath@gmail.com 